\title{
Plasmid DNA containing multiple CpG motifs triggers a strong immune response to hepatitis $B$ surface antigen when combined with incomplete Freund's adjuvant but not aluminum hydroxide
}

\author{
ZICHAO LUO ${ }^{1,2}$, HUASHAN SHI ${ }^{1}$, HAILONG ZHANG ${ }^{1}$, MENG LI ${ }^{1}$, YUWEI ZHAO ${ }^{1}$, JING ZHANG ${ }^{1}$, \\ FUCHUN GUO $^{1}$, SHAN LUO ${ }^{1}$, PING SUN ${ }^{1}$, DONGMEI ZHANG ${ }^{1}$, ZHIYONG QIAN ${ }^{1}$ and LI YANG ${ }^{1}$ \\ ${ }^{1}$ State Key Laboratory of Biotherapy and Cancer Center, West China Hospital, West China Medical School, \\ Sichuan University, Chengdu, Sichuan 610041; ${ }^{2}$ CAS/CUHK Research Centre for Biosensors and Medical Instruments, \\ Institute of Biomedical and Health Engineering, Shenzhen Institutes of Advanced Technology \\ Chinese Academy of Sciences, Shenzhen, Guangdong 518005, P.R. China
}

Received February 17, 2012; Accepted July 4, 2012

DOI: $10.3892 / \mathrm{mmr} .2012 .1079$

\begin{abstract}
Adjuvants are important components of recombinant protein vaccines which are often poorly immunogenic. For decades, the search for new vaccine adjuvants has been predominantly empirical. In addition, combinations of more than one adjuvant plus antigen have been systematically studied. Plasmid DNA containing additional oligodeoxynucleotides with unmethylated $\mathrm{CpG}$ motifs (CpG ODN) entrapped in liposomes has been used as an adjuvant for DNA vaccines and has shown powerful immunostimulatory functions. In our study, the combination of plasmid DNA containing 16 additional $\mathrm{CpG}$ ODNs (pv-16CpG) and aluminum hydroxide (AL) or incomplete Freund's adjuvant (IFA) was used as an adjuvant for a hepatitis B surface antigen (HBsAg) vaccine to immunize C57BL/6J mice. ELISA and ELISPOT assays were used to analyze the immunological effects of the novel vaccine. A significant enhancement of the anti-HBs titer and seroconversion was observed when the $\mathrm{CpG}$ plasmid was combined with IFA, but not with AL. In addition, anti-HBs antibody isotype analysis revealed that the combination of CpG plasmid and IFA induced a strong HBsAg-specific IgG2a response. Moreover, the ELISPOT assays suggested that pv-16CpG suspended in IFA evoked a strong $\mathrm{T}$ helper 1 (Th1) immune response and high IFN- $\gamma$ production. These results demonstrate that $\mathrm{pv}-16 \mathrm{CpG}$ suspended in IFA is able to
\end{abstract}

Correspondence to: Professor Li Yang, State Key Laboratory of Biotherapy and Cancer Center, West China Hospital, West China Medical School, Sichuan University, 1 Keyuan 4th Road, Gaopeng Street, High Technological Zone, Chengdu, Sichuan 610041, P.R. China

E-mail: yl.tracy73@gmail.com

Key words: adjuvant, alum, incomplete Freund's adjuvant, unmethylated CpG motifs, plasmid DNA induce cellular and humoral immune responses to HBsAg, and confirm its potential as an adjuvant for use in protein vaccines.

\section{Introduction}

Vaccination is an effective method of decreasing the incidence of infectious diseases caused by pathogens to which individuals are susceptible in early life (1). Unlike live and attenuated organism vaccines, the inactive or dead organism vaccines and recombinant protein or subunit vaccines are generally administered with an adjuvant to aid in or enhance the immune response (2-3). Adjuvants are compounds that enhance the ability of the co-inoculated antigens to elicit an early, high and long-lasting immune response with a reduced amount of antigen (4). Owing to the highly purified recombinant proteins, which are increasingly used in the new generation vaccines but are poorly immunogenic, formulations comprising more than one adjuvant have been widely studied in various vaccines. To enhance the potency of the first generation adjuvants, including aluminum salts (also known as alums), emulsions, polymeric particles and liposomes, additional components have been added to them, generating the second generation of vaccine adjuvants. Furthermore, the second generation adjuvants have been licensed in approved vaccine products in the EU, including Pandemrix (AS03; o/w emulsion $+\alpha$-tocopherol) and Fendrix or Cervarix (AS04; MPL + aluminum hydroxide) (5).

Bacterial DNA and oligodeoxynucleotides containing unmethylated $\mathrm{CpG}$ motifs ( $\mathrm{CpG}$ ODN) have been studied as vaccine adjuvants for the induction of protective immunity against bacterial, viral and parasitic infections in numerous animal models (6-9). Previous studies have found that $\mathrm{CpG}$ ODN is able to activate a $\mathrm{T}$ helper 1 (Th1)-like humoral immune response with the production of IgG2a antibodies and a cell-mediated immune response with the secretion of IFN- $\gamma$ cytokines (10-11). Furthermore, another study has shown that plasmid DNA directly injected into the muscle cells of mice elicited potent humoral and cellular immune responses (12). In addition, it has been shown that plasmid DNA may be useful as an adjuvant for DNA vaccines $(13,14)$. Recently, our 
laboratory revealed that plasmid DNA (pcDNA 3.1) encapsulated in cationic liposomes delivered an adjuvant effect in the cancer immunotherapy of murine colon carcinoma (15). Other studies have demonstrated that the adjuvant properties of plasmid DNA were dependent on its content of CpG motifs which interacted with TLR9 and directly induced the production of IL-12 and IFN- $\alpha$ by dendritic cells (16-19). As the plasmid pcDNA 3.1 contains the ampicillin resistance gene, it is commonly used for studies in mice. Considering that the plasmid vector pVAX1, which contains a kanamycin resistance gene, is permitted to be used in humans (20), and the high cost of synthesis of $\mathrm{CpG}$ ODN, we constructed a multiple $\mathrm{CpG}$ plasmid (pv-16CpG) containing 16 CpG motifs (CpG 7909) to examine its adjuvant effect in a hepatitis B surface antigen (HBsAg) vaccine when combined with aluminum hydroxide (AL) or incomplete Freund's adjuvant (IFA).

Aluminium salts, particularly phosphate or hydroxide salts, have been widely used in human and veterinary vaccines since 1930 with an excellent record of safety (21). Aluminum salts markedly induce the generation of Th2 cytokines (IL-4 and IL-5) and antibodies of the IgG1 isotype (22-23). IFA, a waterin-oil emulsion, has been widely used in veterinary vaccine products and tested in humans (24-25). The main mechanisms of action of aluminum salts and IFA are similar, they prolong the duration of antigen persistence at the injection site (5).

Since the introduction of a hepatitis B vaccine containing alum over 30 years ago, a decline in the global incidence of HBV infection has been observed (26). However, 5-10\% of healthy immunocompent individuals fail to produce a protective antibody response to the HBV vaccine (27). Improving the immunogenicity of the HBV vaccine is a strategy that can be employed to overcome this problem. Therefore, new adjuvants that elicit strong immune responses to HBsAg are required. Previous studies have shown that certain adjuvant combinations induce a stronger immune response than either individual component alone (28). In this study, we used formulations of pv-16CpG with AL or IFA as adjuvants for a hepatitis B vaccine. The results showed that pv-16CpG suspended in IFA triggered a stronger immune response against $\mathrm{HBsAg}$ in $\mathrm{C} 57 \mathrm{BL} / 6 \mathrm{~J}$ mice than $\mathrm{pv}-16 \mathrm{CpG}$ combined with AL. The plasmid pv-16CpG suspended in IFA induced a Th1 immune response to $\mathrm{HBsAg}$, characterized by the generation of specific IgG2a antibodies and IFN- $\gamma$ in antigen stimulated $\mathrm{T}$ cells. We suggest that $\mathrm{pv}-16 \mathrm{CpG}$, which may facilitate the manufacturing process, may be used as an adjuvant for $\mathrm{HBsAg}$ vaccines when mixed with IFA.

\section{Materials and methods}

Plasmid $p v-16 C p G$ preparation. The plasmid pv-16CpG was constructed as follows: $16 \mathrm{CpG}$ motif ODN (16 x 5'-TCGTCGTTTTGTCGTTTTGTCGTT-3') fragments were synthesized and inserted into the plasmid pVAX1 (Invitrogen Life Technologies, San Diego, CA, USA) using the enzymes HindIII (5'-end of the fragment) and BamHI (3'-end of the fragment) from Invitrogen. The fragment sequences were confirmed by DNA sequencing. The plasmid pv-16CpG was prepared using the Endofree Plasmid Giga kit (Qiagen, Chatsworth, CA, USA). There was no genomic DNA, small DNA fragments or RNA in the pv-16CpG DNA $\left(\mathrm{OD}_{260 / 2280}=1.8-2.0\right)$.

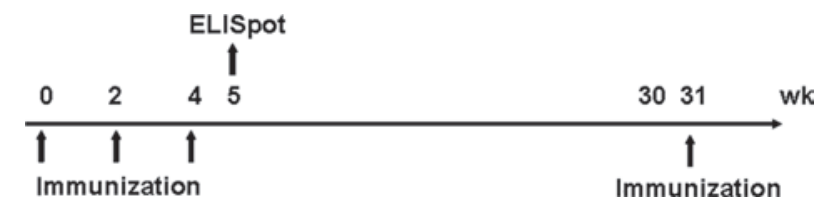

Figure 1. Schema of the immunization protocol. Mice $(\mathrm{n}=8)$ were immunized with $\mathrm{HBsAg}(0.1 \mu \mathrm{g})$ and $\mathrm{AL}\left(25 \mu \mathrm{g} \mathrm{A} 1^{3+}\right)$, IFA $(50 \mu \mathrm{l} ; \mathrm{v} / \mathrm{v}=1: 1)$, $\mathrm{AL}+\operatorname{pvAX}(50 \mu \mathrm{g}), \mathrm{IFA}+\operatorname{pvAX}(50 \mu \mathrm{g}), \mathrm{AL}+\operatorname{pv}-16 \mathrm{CpG}(50 \mu \mathrm{g})$ or IFA + pv-16CpG $(50 \mu \mathrm{g})$ in weeks 0,2 and 4 . In addition, mice were immunized with the same dose of vaccine in week 31 . Following the third immunization, mice $(n=3)$ were sacrificed and an ELISPOT assay was performed. Sera were collected in weeks 1, 3, 5, 7, 9, 13, 17, 21, 25, 30, 32, 34 and 50, and the anti-HBsAg titers were measured by endpoint dilution ELISA. HBsAg, hepatitis B surface antigen; AL, aluminum hydroxide; IFA, incomplete Freund's adjuvant; pvAX, pv-16CpG, plasmid vectors; ELISA, enzyme-linked immunosorbent assay.

Reagents. AL gel adjuvant was purchased from Brenntag Biosector (Frederikssund, Denmark) and IFA was purchased from Sigma Poole (Dorset, UK). The hepatitis B surface antigen (subtype ad) protein was purchased from American Research Products, Inc. (Belmont, MA, USA).

Animals and immunizations. Six to eight-week-old female C57BL/6J mice ( $\mathrm{n}=8$ mice per group) were purchased from the Experimental Animal Center of Sichuan University and were housed in our animal research facility. The mice were immunized intramuscularly (hind limbs) three times at 2-week intervals (in weeks 0, 2 and 4) with $0.1 \mu \mathrm{g}$ HBsAg and adjuvant in a total volume of $100 \mu \mathrm{l}$ (Fig. 1). The adjuvant amounts used for each immunization were: $25 \mu \mathrm{g} \mathrm{Al}^{3+}, 50 \mu \mathrm{g}$ pvAX, $50 \mu \mathrm{g}$ pv-16CpG or IFA $50 \mu \mathrm{l}(\mathrm{v} / \mathrm{v}$ 1:1), mixed by syringe. The mice were administered a booster immunization with the same dose in week 31. Blood samples were collected from the animals in weeks $0,1,3,5,7,9,11,13,17,21,25,30,32,34$ and 50 following the immunization. The serum was divided into aliquots and was maintained at $-70^{\circ} \mathrm{C}$.

Enzyme-linked immunosorbent assay (ELISA). To measure the HBsAg-specific antibody response, a 96-well plate was coated overnight at $4^{\circ} \mathrm{C}$ with $100 \mu \mathrm{l} \mathrm{HBsAg}$ solution $(1 \mu \mathrm{g} / \mathrm{ml})$ in sodium carbonate buffer. The plate was washed three times with $0.1 \%$ Tween in PBS (PBST), and then blocked with 5\% skimmed milk in PBST for $1 \mathrm{~h}$ at $37^{\circ} \mathrm{C}$. After being washed 5 times with PBST, the plate was incubated with serially diluted mice sera in $5 \%$ skimmed milk for $1 \mathrm{~h}$ at $37^{\circ} \mathrm{C}$, followed by further washes. Bound antibodies were detected using horseradish peroxidase-conjugated goat anti-mouse IgG, IgG1 IgG2a, IgG2b or IgG3 (Southern Biotechnologies, Birmingham, AL, USA) diluted at $1: 400$ for $1 \mathrm{~h}$ at $37^{\circ} \mathrm{C}$. The plate was washed again and incubated with $100 \mu \mathrm{l}$ substrate-chromogen solution (SureBlue ${ }^{\mathrm{TM}}$ TMB Microwell Peroxidase substrate, KPL, Inc., Gaithersburg, MD, USA) for $20 \mathrm{~min}$ at room temperature. A volume of $0.5 \mathrm{M} \mathrm{H}_{2} \mathrm{SO}_{4}$ (100 $\mu \mathrm{l})$ was added to terminate the reaction and the absorbance was read at $450 \mathrm{~nm}$ using a Bio-Rad (Hercules, CA, USA) microtiter plate reader. ELISA titers were expressed as the reciprocal of the highest dilution resulting in a value of two standard deviations above that of non-immunized serum with a cut-off of 0.2 . 
IFN- $\gamma$ and IL-4 ELISPOT assays. To measure the numbers of IFN- $\gamma$ and IL-4 secreting cells, the dual-color ELISPOT Mouse IFN- $\gamma /$ IL-4 kit (R\&D systems, Inc., Minneapolis, MN, USA) was used. Following the third immunization, the mice were sacrificed and their splenocytes were separated as described in the manual of the EZ-sep ${ }^{\mathrm{TM}}$ mouse 1X. (Dakewe Biotech Co., Ltd., Shenzhen, China). The splenocytes were added to triplicate wells at a concentration of $5 \times 10^{5}$ cells/well and cultured in the presence of HBsAg $(5 \mu \mathrm{g} / \mathrm{ml})$ or ConA $(1 \mu \mathrm{g} / \mathrm{ml})$ for $48 \mathrm{~h}$. Stained spots were counted using a computer-assisted ELISPOT image analyzer (Immunospot, Cellular Technology Ltd., Cleveland, OH, USA).

Electrophoretic mobility shift assay (EMSA) and scanning electron microscopy (SEM) experiments. Binding assays were performed by incubating pv-16CpG $(10 \mu \mathrm{g})$ with various amounts of AL in a final volume of $100 \mu 1$ of $10 \mathrm{mM}$ Tris- $\mathrm{HCl}$ and $270 \mathrm{mM}$ sorbitol ( $\mathrm{pH} 7.4)$. After incubating at $37^{\circ} \mathrm{C}$ for $10 \mathrm{~min}$, the complexes were centrifuged at $3000 \mathrm{rpm}$ for $5 \mathrm{~min}$. The deposits were subjected to electrophoresis through a $0.8 \%$ agarose gel at $120 \mathrm{~V}$ for $15 \mathrm{~min}$. The DNA was visualized by ethidium bromide staining. The SEM experiments were performed using a JSM-5900LV SEM instrument to inspect the sizes of the particles. The samples were prepared according to the prescription of administration for mice and were then gold-coated and observed under an accelerating voltage of $20 \mathrm{kV}$.

Statistical analysis. The studies were performed on groups of 3-5 individual mice. Statistical comparisons were carried out using one-way analysis of variance (ANOVA) followed by Tukey's test. Results were considered statistically significant at $\mathrm{p}<0.05$.

\section{Results}

Seroconversion induced by IFA + pv-16CpG. In week 1 following the immunization, $20 \%$ of mice in the $\mathrm{AL}+$ pvAX, IFA + pvAX and IFA + pv-16CpG groups had produced anti-HBs (titer $\geq 100$ ) (Fig. 2). In week 3 post-immunization, the anti-HBs seroconversion rate of the $\mathrm{AL}$ and $\mathrm{AL}+\mathrm{pvAX}$ groups had also reached $20 \%$. In addition, at this time, the rates in the IFA + pvAX and IFA + pv-16CpG groups were 40 and $80 \%$, respectively. However, no seroconversion was identified in the $\mathrm{AL}+\mathrm{pv}-16 \mathrm{CpG}$ and IFA groups. The seroconversion rate in the IFA + pv-16CpG group was clearly improved compared with the other 5 groups. However, 5 weeks after immunization, the seroconversion rates in the $\mathrm{AL}+\mathrm{pv}-16 \mathrm{CpG}$ and IFA groups were 40 and $20 \%$, respectively. Moreover, while the seroconversion rate of the $\mathrm{AL}+\mathrm{pvAX}$ group reached $60 \%$, the rates in the other 3 groups were $100 \%$.

Enhancement of anti-HBs titer by IFA + pv-16CpG. The kinetics of the HBsAg-specific humoral immune responses were monitored over the 50 weeks following immunization. C57BL/6J mice were immunized by intramuscular (i.m.) injection of $0.1 \mu \mathrm{g} \mathrm{HBsAg}$ with various adjuvants at different time points (Fig. 2). When the double adjuvant combinations were used, the anti-HBs titer of the IFA + pv- $16 \mathrm{CpG}$ group in week 13 (first peak) was 50 -fold higher than that of the IFA

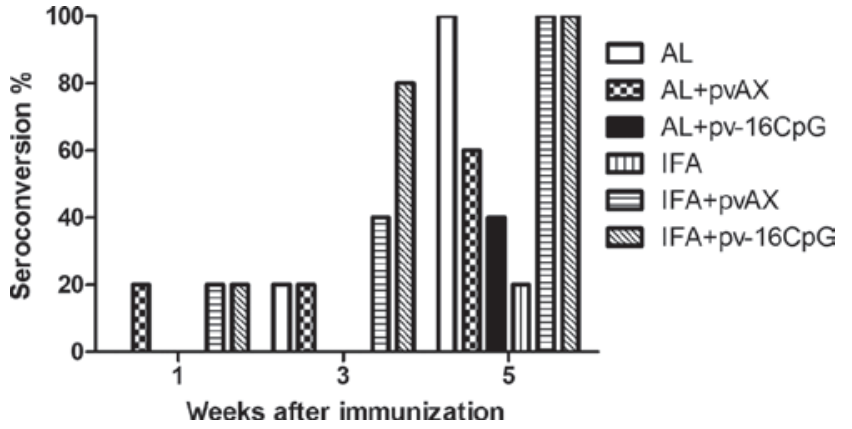

Figure 2. Percentage of seroconversion for C57BL/6J mice immunized in weeks 0, 2 and 4 using HBsAg with various adjuvants. C57BL/6J mice $(n=5)$ were immunized 3 times by intramuscular (i.m.) injection with $0.1 \mu \mathrm{g}$ HBsAg formulated with $25 \mu \mathrm{g}$ AL, IFA $(50 \mu \mathrm{l} ; \mathrm{v} / \mathrm{v}=1: 1), \mathrm{AL}+\mathrm{pvAX}$ $(50 \mu \mathrm{g}), \mathrm{IFA}+\operatorname{pvAX}(50 \mu \mathrm{g}), 25 \mu \mathrm{g} \mathrm{AL}+50 \mu \mathrm{g} \mathrm{pv}-16 \mathrm{CpG}$, or $50 \mu \mathrm{g}$ IFA + pv-16CpG $(50 \mu 1)$. Sera obtained in weeks 1,3 and 5 were assayed for anti-HBsAg by endpoint-dilution ELISA assay. Seroconversion was defined as a dilution titer $\geq 100$ with a cut-off value of 0.2 . HBsAg, hepatitis B surface antigen; AL, aluminum hydroxide; IFA, incomplete Freund's adjuvant; pvAX, pv-16CpG, plasmid vectors; ELISA, enzyme-linked immunosorbent assay.

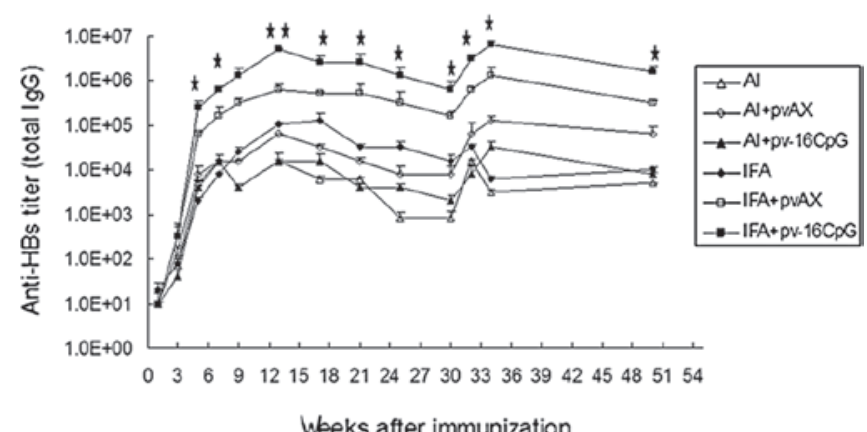

Figure 3. IgG antibody titer kinetics against HBsAg in C57BL/6J mice immunized with AL, IFA, AL + pvAX, IFA + pvAX, AL + pv-16CpG or IFA + pv-16CpG. Mice were immunized as described above. ELISA titers are defined as the reciprocal of the highest dilution resulting in a reading of two standard deviations above that of non-immunized mice with a cut-off value of 0.2. Sera obtained from each mouse were used to detect the titer by ELISA in each group. Data are presented as the group mean for total IgG titer to HBsAg. Vertical lines are the SEM. Two other experiments produced similar results; ${ }^{\star} \mathrm{p}<0.05 ;{ }^{\star \star} \mathrm{p}<0.001$. HBsAg, hepatitis B surface antigen; AL, aluminum hydroxide; IFA, incomplete Freund's adjuvant; pvAX, pv-16CpG, plasmid vectors; ELISA, enzyme-linked immunosorbent assay.

group and 320-fold higher than that of the AL group (Fig. 3). However, there was no increase in the anti-HBs titers when the combination of $\mathrm{AL}$ and $\mathrm{pv}-16 \mathrm{CpG}$ was used as the adjuvant. The IFA + pvAX group underwent a significant increase compared with the remaining groups, with the exception of the IFA + pv-16CpG group. Throughout the study, the titers of the IFA + pv-16CpG group were 5-8-fold higher than that of the IFA + pvAX group at 50 weeks. The antibody titer of the IFA group underwent a sustained growth following the first immunization, and this increasing trend stopped in week 17, while in the other groups, it stopped in week 13. Twenty-one weeks after immunization, the titers of the 6 groups exhibited a sustained decrease. To detect the memory response evoked by the vaccine, the mice were boosted with the same dose of vaccine in week 31. A 2- to 20-fold increase in anti-HBs titers was observed in all the groups. However, the anti-HBs titer of the IFA + pv-16CpG group in week 32 was 
A

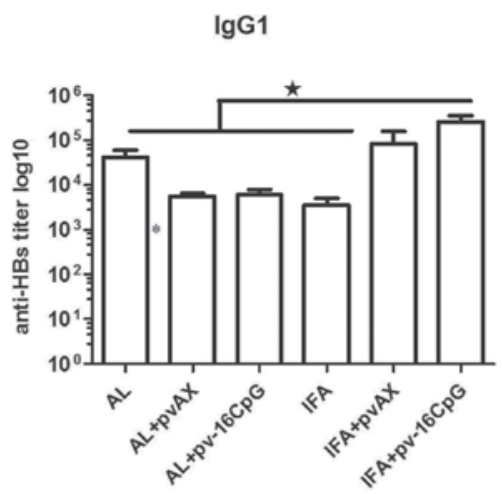

C

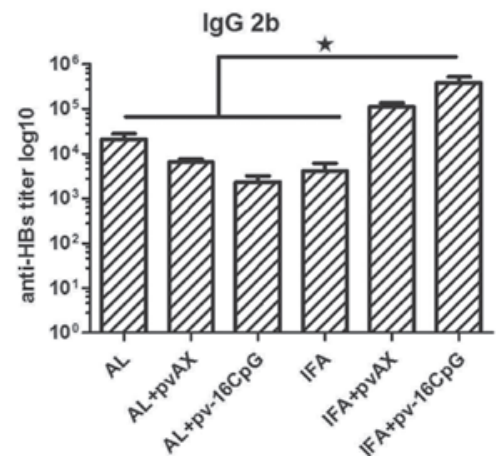

B

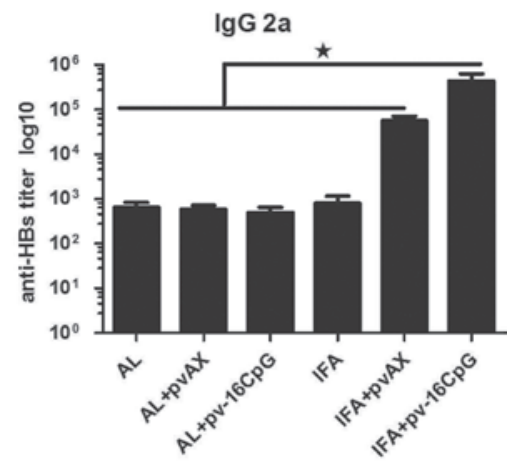

$\operatorname{lgg} 3$

D

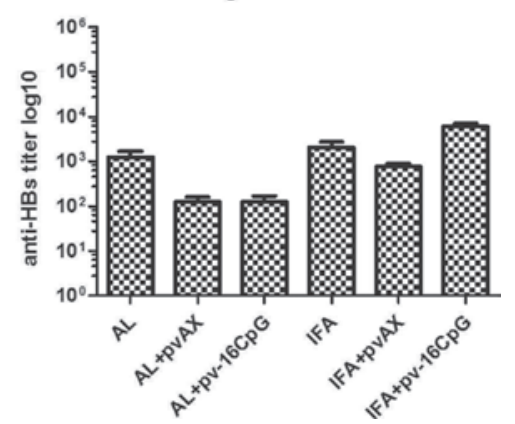

Figure 4. Antibody isotypes in C57BL/6J mice 7 weeks after immunization with various vaccines. Anti-HBs serum IgG isotype titers were determined by end-point dilution ELISA assay. Data are presented as the mean \pm SEM of ELISA titers obtained from the sera of 5 animals. Each graph is representative of at least three experiments. ELISA, enzyme-linked immunosorbent assay; AL, aluminum hydroxide; pvAX, pV-16CpG, plasmid vectors; IFA, incomplete Freund's adjuvant.

40- or 300-fold higher than that in the AL and IFA groups, respectively. Furthermore, the anti-HBs antibody titer in the IFA + pv-16CpG group in week 32 was 800 -fold higher than that in the $\mathrm{AL}+\mathrm{pv}-16 \mathrm{CpG}$ group (Fig. 3).

Antibody isotype profiles evoked by IFA $+p v-16 C p G$. The ratio of IgG2a to IgG1 antibody levels is often used to evaluate the type of Th response, with a value $>1$ indicating a predominantly Th1 response and $<1$ indicating a predominantly Th2 response. Immunization with AL or IFA alone generated a Th2 response, with higher levels of anti-HBs antibodies of the IgG1 isotype than those of the $\mathrm{IgG} 2 \mathrm{a}$ isotype (Fig. 4A and $\mathrm{B}$ ). The values of $\operatorname{IgG} 2 \mathrm{a} / \mathrm{IgG} 1$ were 0.021 and 0.25 in the $\mathrm{AL}$ and IFA groups, respectively, 7 weeks after immunization. When the plasmid pv-16CpG combined with AL was added as the adjuvant, the anti-HBs titers of the IgG1 and IgG2a isotypes were lower than those in the AL group. In addition, the $\mathrm{IgG} 2 \mathrm{a} / \mathrm{IgG} 1$ ratio was $0.063,7$ weeks after immunization. When the plasmid pvAX was combined with AL or IFA, the values of $\mathrm{IgG} 2 \mathrm{a} / \mathrm{IgG} 1$ in week 7 were 0.078 and 0.5 , respectively. This finding suggested that the combination of plasmid and AL or IFA induced a Th2 immune response. However, with an increased effect on IgG1 and IgG 2 a antibody titers, the combination of plasmid pv-16CpG and IFA evoked a Th1 response (Fig. 4A and B) with a value of $\mathrm{IgG} 2 \mathrm{a} / \mathrm{IgG1}>1$ (2.0). Moreover, the $\operatorname{IgG} 2 \mathrm{~b}$ and $\operatorname{IgG} 3$ antibody titers in the IFA + pv-16CpG group increased significantly compared with those in the IFA group, but decreased in the $\mathrm{AL}+$ pv-16CpG group when compared with the AL group (Fig. 4C and D). These findings suggest that the plasmid pv-16CpG has great

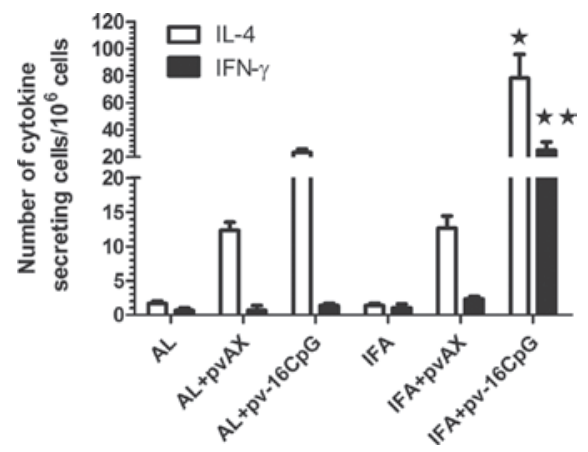

Figure 5. Frequency of HBsAg-specific IFN- $\gamma$ - and IL-4-secreting cells in splenocytes of mice 5 weeks after immunization. C57BL/6J mice $(n=3)$ were immunized with $0.1 \mu \mathrm{g}$ HBsAg combined with AL, IFA, AL + pv-16CpG, IFA + pv-16CpG, AL + pvAX or IFA + pvAX in weeks 0,2 and 4 . The number of cytokine-secreting cells were determined by a computer-assisted ELISPOT image analyzer. Bars show the mean \pm SEM number of cytokinespecific cells in splenocytes as determined by the ELISPOT assay. ${ }^{\star} \mathrm{p}<0.05$; ${ }^{\star \star} \mathrm{p}<0.001$. HBsAg, hepatitis B surface antigen; AL, aluminum hydroxide; IFA, incomplete Freund's adjuvant; pv-16CpG, pvAX, plasmid DNA.

potential for improving the immune response when combined with IFA but not with AL.

Assay of HBsAg-specific T cell immune response by ELISPOT. To characterize the immune responses induced by the various formulations, the production of IL- 4 and IFN- $\gamma$ by HBsAgexposed splenocytes from immunized mice were examined by re-stimulating the splenocytes with HBsAg. The number of IL-4-secreting $\mathrm{T}$ cells in the mice immunized with 


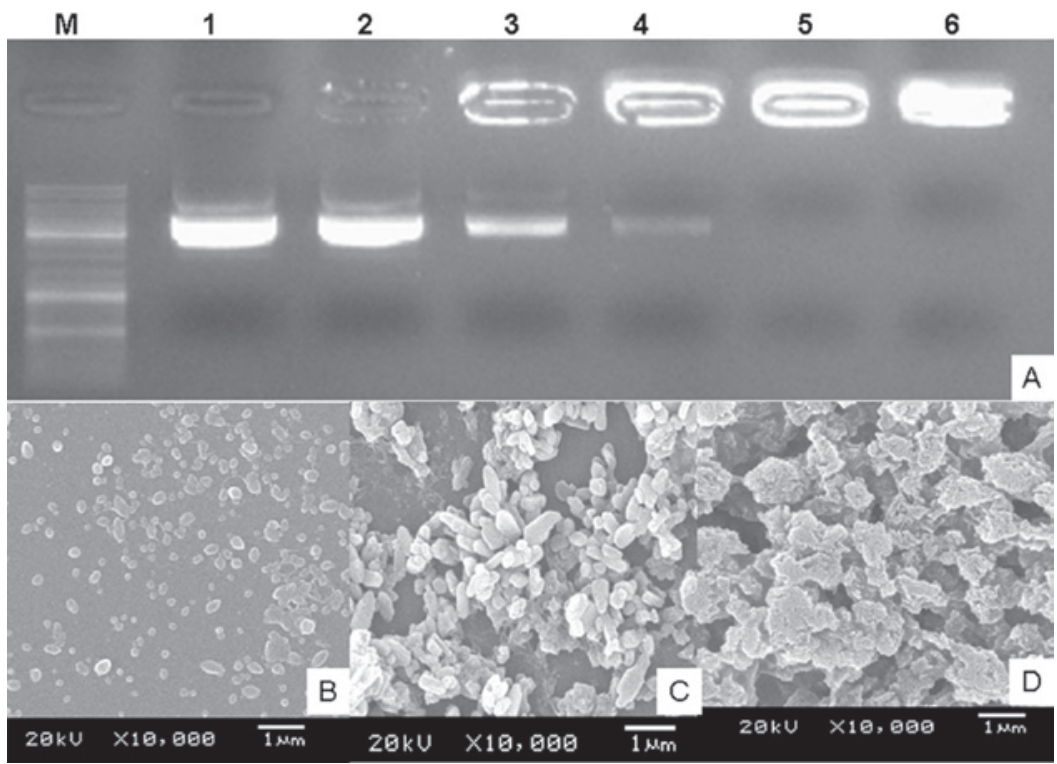

Figure 6. Electrophoretic mobility shift assay (EMSA) and scanning electron microscopy (SEM) of AL and pvAX or pv-16CpG complexes. (A) EMSA of $\mathrm{AL}$ and pv-16CpG complex: M, marker; lane 1, pv-16CpG (10 $\mu \mathrm{g})$; lane 2, pv-16CpG (10 $\mu \mathrm{g})+\mathrm{AL}(5 \mu \mathrm{g})$; lane 3, pv-16CpG $(10 \mu \mathrm{g})+\mathrm{AL}(10 \mu \mathrm{g}) ; 1 a n e-4$, pv-16CpG $(10 \mu \mathrm{g})+\mathrm{AL}(20 \mu \mathrm{g})$; lane 5, pv-16CpG $(10 \mu \mathrm{g})+\mathrm{AL}(40 \mu \mathrm{g})$; lane 6, pv-16CpG $(10 \mu \mathrm{g})+\mathrm{AL}(80 \mu \mathrm{g})$. (B) SEM micrograph of AL $(25 \mu \mathrm{g})$ diluted and vortexed in a total volume of $100 \mu \mathrm{l}$. (C) SEM micrograph of AL and pvAX complex: AL ( $25 \mu \mathrm{g})$ and pvAX (50 $\mu \mathrm{g})$ diluted and vortexed in a total volume of $100 \mu 1$. (D) SEM micrograph of AL and pv-16CpG complex: AL (25 $\mu \mathrm{g})$ and pv-16CpG $(50 \mu \mathrm{g})$ diluted and vortexed in a total volume of $100 \mu 1$. Each graph is representative of at least three experiments. $\mathrm{AL}$, aluminum hydroxide; pvAX, pv-16CpG, plasmid vectors.

$\mathrm{AL}+\mathrm{pv}-16 \mathrm{CpG}$ or IFA + pv-16CpG were significantly higher than that in the AL, AL + pvAX, IFA or IFA + pvAX groups (Fig. 5). The number of IL-4-secreting splenocytes in the mice immunized with $\mathrm{AL}+\mathrm{pv}-16 \mathrm{CpG}$ or IFA $+\mathrm{pv}-16 \mathrm{CpG}$ were approximately 15 - and 30 -fold higher than in the mice immunized with AL or IFA alone, respectively (Fig. 5). When the plasmid pvAX was added to AL or IFA, the number of IL-4-secreting splenocytes was also lower than that in the IFA + pv-16CpG group $(\mathrm{p}<0.05)$. Furthermore, the highest number of IFN- $\gamma$-secreting $\mathrm{T}$ cells was found in the mice receiving IFA + pv-16CpG. The number of IFN- $\gamma$-secreting $\mathrm{T}$ cells in the mice receiving IFA + pv-16CpG was almost 30 -fold higher than that in the mice from the other groups $(\mathrm{p}<0.001)$. Moreover, the higher IFN- $\gamma$ secretion indicated a potent type 1 cell-mediated immune response.

\section{Discussion}

Vaccines are widely used in the protection and eradication of a wide range of diseases, including smallpox, polio, measles, whooping cough and hepatitis. Sub-unit vaccines, including HBsAg-based vaccines, have a good record of safety but low efficacy in eliciting an immune response (29). Therefore, adjuvants which enhance the immunogenicity of these vaccines are urgently required.

Aluminum salts (particularly aluminum hydroxide or phosphate), which have been licensed as adjuvants for human use, have been widely and successfully used in a great number of licensed vaccines although they induce a poor cytotoxic $\mathrm{T}$ cell and Th1 immune response (30-31). As shown in Figs. 4 and 5, AL induces a Th2 immune response, with predominantly IgG1 antibody and IL-4 secretion from immunized splenocytes. When the plasmid pv-16CpG was combined with AL, it induced a weaker immune response than AL alone.
IFA, a water-in-oil emulsion adjuvant, which produces a high and sustained antibody response, has been used in experimental vaccines in mice, rabbits, guinea pigs and rhesus macaques (24). Furthermore, IFA induces a Th2 type response, which is not effective for a cellular immune response and limits the adjuvant activity of IFA. Our results showed that when IFA was used alone, it induced predominantly IgG1 antibody (Fig. 4A and B) and IL-4 production, as detected by the IL-4/IFN- $\gamma$ ELISPOT assay (Fig. 5). However, mixing or using IFA in conjunction with other adjuvant-active compounds has been successful in animal testing and preclinical trials (24). Furthermore, findings of another study have shown that insect DNA and CpG suspended in IFA was a more powerful adjuvant in mice than CFA, which is considered as the 'gold standard' for adjuvant function (32). In our study, the use of a combination of IFA and pv-16CpG as the adjuvant for a HBsAg vaccine, resulted in higher anti-HBs antibody titers than the other adjuvants tested (at least 10-fold higher than the other groups throughout the experiment; Fig. 3) and significantly increased IFN- $\gamma$ production, even compared with the $\mathrm{AL}+\mathrm{pv}-16 \mathrm{CpG}$ group $(\mathrm{p}<0.001)$. The booster immunization in week 31 resulted in a profoundly increased and sustained memory response in the IFA + pv- $16 \mathrm{CpG}$ group. The data suggest that pv-16CpG suspended in IFA evokes cellular and humoral immune responses.

The data demonstrated that pv-16CpG exhibited a strong adjuvant activity when combined with IFA but not with AL. The reasons which may explain this result include the fact that the mechanism of action of adjuvant aluminum salts involves the formation of an antigen depot at the injection site (33), the electrostatic attractive forces between the aluminum salt (positive charge) and antigen (negative charge) (34). As pv-16CpG itself may be considered as a polyelectrolyte with a high negative charge (35), there is likely to be an electrostatic interaction 
between AL and pv-16CpG. As shown in Fig. 6, pv-16CpG was completely adsorbed by AL when the ratio was $1: 10$ by weight. This interaction may reduce the ability of the aluminum salt to form an antigen depot from which the antigen is slowly released. However, there may be not an electrostatic interaction between pv-16CpG and IFA. Moreover, in the microdroplets of oil formed by mixing, the electrostatic attractive force between pv-16CpG and the HBsAg protein may contribute to the slow release of antigen. Due to the limitations of the technology, EMSA could not be performed for IFA and plasmid DNA.

Another explanation for our results is that the size of the antigen or the antigen formulated with an adjuvant is a significant factor for its efficient uptake by antigen-presenting cells (APCs). The range of particle sizes which allows the most efficient uptake by APCs is $10-3,000 \mathrm{~nm}$. In addition, the average particle sizes of emulsions (such as IFA) and mineral salts (such as AL) are 200-8,000 and 800-12,000 nm, respectively (36). The particle size of AL is smaller than $1 \mu \mathrm{m}$, but becomes larger than $1.5 \mu \mathrm{m}$ when $\mathrm{pv}-16 \mathrm{CpG}$ is combined with it (Fig. 6B and C), and this change may affect the take up of the antigen by APCs. The mechanism of adjuvant activity in the vaccine comprising pv-16CpG suspended in IFA requires further study.

In conclusion, the results of our studies have shown that pv-16CpG may be used as an adjuvant for HBsAg vaccines when combined with IFA but not with AL, and evoked cellular and humoral immune responses against $\mathrm{HBsAg}$.

\section{Acknowledgements}

This study is supported by the Program for New Century Excellent Talents in University (NCET) (NCET-09-0575) and the National Natural Science Foundation of China (grant no. 31070815).

\section{References}

1. Weeratna RD, McCluskie MJ, Xu Y and Davis HL: CpG DNA induces stronger immune responses with less toxicity than other adjuvants. Vaccine 18: 1755-1762, 2000.

2. Ulmer JB, Valley U and Rappuoli R: Vaccine manufacturing: challenges and solutions. Nat Biotechnol 24: 1377-1383, 2006.

3. Aguilar JC and Rodriguez EG: Vaccine adjuvants revisited. Vaccine 25: 3752-3762, 2007.

4. Gupta RK and Siber GR: Adjuvants for human vaccines-current status, problems and future prospects. Vaccine 13: 1263-1276, 1995.

5. O'Hagan DT and De Gregorio E: The path to a successful vaccine adjuvant - 'the long and winding road'. Drug Discov Today 14: 541-551, 2009.

6. Stacey KJ and Blackwell JM: Immunostimulatory DNA as an adjuvant in vaccination against Leishmania major. Infect Immun 67: 3719-3726, 1999.

7. Jones TR, Obaldia N III, Gramzinski RA, et al: Synthetic oligodeoxynucleotides containing $\mathrm{CpG}$ motifs enhance immunogenicity of a peptide malaria vaccine in Aotus monkeys. Vaccine 17: 3065-3071, 1999.

8. Elkins KL, Rhinehart-Jones TR, Stibitz S, Conover JS and Klinman DM: Bacterial DNA containing CpG motifs stimulates lymphocyte-dependent protection of mice against lethal infection with intracellular bacteria. J Immunol 162: 2291-2298, 1999.

9. Moldoveanu Z, Love-Homan L, Huang WQ and Krieg AM: CpG DNA, a novel immune enhancer for systemic and mucosal immunization with influenza virus. Vaccine 16: 1216-1224, 1998

10. Klinman DM, Yi AK, Beaucage SL, Conover J and Krieg AM: CpG motifs present in bacteria DNA rapidly induce lymphocytes to secrete interleukin 6, interleukin 12, and interferon gamma. Proc Natl Acad Sci USA 93: 2879-2883, 1996.
11. Brazolot Millan CL, Weeratna R, Krieg AM, Siegrist CA and Davis HL: CpG DNA can induce strong Th1 humoral and cellmediated immune responses against hepatitis B surface antigen in young mice. Proc Natl Acad Sci USA 95: 15553-15558, 1998.

12. Corr M, Lee DJ, Carson DA and Tighe H: Gene vaccination with naked plasmid DNA: mechanism of CTL priming. J Exp Med 184: 1555-1560, 1996.

13. Kojima Y, Xin KQ, Ooki T, et al: Adjuvant effect of multi-CpG motifs on an HIV-1 DNA vaccine. Vaccine 20: 2857-2865, 2002.

14. Klinman DM, Barnhart KM and Conover J: CpG motifs as immune adjuvants. Vaccine 17: 19-25, 1999.

15. Zhang XP, Yang L, Shi HS, et al: An N-, C-terminally truncated basic fibroblast growth factor and LPD (liposome-polycationDNA) complexes elicits a protective immune response against murine colon carcinoma. Cancer Biol Ther 10: 276-281, 2010.

16. Wagner H: Bacterial CpG DNA activates immune cells to signal infectious danger. Adv Immunol 73: 329-368, 1999.

17. Krieg AM: $\mathrm{CpG}$ motifs in bacterial DNA and their immune effects. Annu Rev Immunol 20: 709-760, 2002.

18. Tudor D, Dubuquoy C, Gaboriau V, Lefèvre F, Charley B and Riffault S: TLR9 pathway is involved in adjuvant effects of plasmid DNA-based vaccines. Vaccine 23: 1258-1264, 2005.

19. Spies B, Hochrein H, Vabulas M, et al: Vaccination with plasmid DNA activates dendritic cells via Toll-like receptor 9 (TLR9) but functions in TLR9-deficient mice. J Immunol 171: 5908-5912, 2003.

20. Gurunathan S, Klinman DM and Seder RA: DNA vaccines: immunology, application, and optimization. Annu Rev Immunol 18: 927-974, 2000.

21. Cox JC and Coulter AR: Adjuvants-a classification and review of their modes of action. Vaccine 15: 248-256, 1997.

22. Weeratna R, Comanita L and Davis HL: CPG ODN allows lower dose of antigen against hepatitis $B$ surface antigen in BALB/c mice. Immunol Cell Biol 81: 59-62, 2003.

23. Mancino D and Ovary Z: Adjuvant effects of amorphous silica and of aluminium hydroxide on IgE and IgG1 antibody production in different inbred mouse strains. Int Arch Allergy Appl Immunol 61: 253-258, 1980.

24. Jensen FC, Savary JR, Diveley JP and Chang JC: Adjuvant activity of incomplete Freund's adjuvant. Adv Drug Deliv Rev 32: 173-186, 1998.

25. Billiau A and Matthys P: Modes of action of Freund's adjuvants in experimental models of autoimmune diseases. J Leukoc Biol 70: 849-860, 2001

26. Liaw YF and Chu CM: Hepatitis B virus infection. Lancet 373: 582-592, 2009.

27. Zuckerman JN: Protective efficacy, immunotherapeutic potential, and safety of hepatitis B vaccines. J Med Virol 78: $169-177,2006$

28. Kovacs-Nolan J, Latimer L, Landi A, et al: The novel adjuvant combination of $\mathrm{CpG}$ ODN, indolicidin and polyphosphazene induces potent antibody- and cell-mediated immune responses in mice. Vaccine 27: 2055-2064, 2009.

29. Perrie Y, Mohammed AR, Kirby DJ, McNeil SE and Bramwell VW: Vaccine adjuvant systems: enhancing the efficacy of sub-unit protein antigens. Int J Pharm 364: 272-280, 2008.

30. Harandi AM, Medaglini D and Shattock RJ; Working Group convened by EUROPRIS: Vaccine adjuvants: a priority for vaccine research. Vaccine 28: 2363-2366, 2010.

31. Lindblad EB: Aluminium compounds for use in vaccines. Immunol Cell Biol 82: 497-505, 2004

32. Sun S, Kishimoto H and Sprent J: DNA as an adjuvant: capacity of insect DNA and synthetic oligodeoxynucleotides to augment T cell responses to specific antigen. J Exp Med 187: 1145-1150, 1998.

33. Shirodkar S, Hutchinson RL, Perry DL, White JL and Hem SL: Aluminum compounds used as adjuvants in vaccines. Pharm Res 7: 1282-1288, 1990.

34. al-Shakhshir RH, Regnier FE, White JL and Hem SL: Contribution of electrostatic and hydrophobic interactions to the adsorption of proteins by aluminium-containing adjuvants. Vaccine 13: 41-44, 1995.

35. Gürsel M, Tunca S, Ozkan M, Ozcengiz G and Alaeddinoglu G: Immunoadjuvant action of plasmid DNA in liposomes. Vaccine 17: 1376-1383, 1999.

36. Bachmann MF and Jennings GT: Vaccine delivery: a matter of size, geometry, kinetics and molecular patterns. Nat Rev Immunol 10: 787-796, 2010. 